\title{
MODELO DE GESTIÓN ESTRATÉGICA DE RESPONSABILIDAD SOCIAL ORGANIZACIONAL
}

\section{STRATEGIC MANAGEMENT MODEL OF CORPORATE SOCIAL RESPONSIBILITY}

\author{
Ana María Díaz Arrieta* \\ Miguel Ángel Bolívar Córdoba* \\ Universidad Simón Bolívar, sede Barranquilla
}

RECIBIDO: Febrero 2 de 2014

ACEPTADO: Abril 8 de 2014

\section{RESUMEN}

El objetivo del presente artículo de investigación consistió en diseñar un modelo de un sistema de gestión, que permitiera mejorar la calidad en una organización, desde la perspectiva de la responsabilidad social. El método utilizado fue el interpretativo analítico, bajo un enfoque mixto y con un corte propositivo. La muestra fue estratificada con 178 elementos (entre empresas y personas naturales). Las técnicas de investigación utilizadas fueron: la observación participante; encuestas y entrevistas a trabajadores, directivos y clientes. El resultado obtenido fue la creación de un modelo de sistema de gestión y de responsabilidad social, basado en la teoría de los stakeholders y fundamentado en la teoría del bien común. Aparte de su utilidad especifica, se concluye que el modelo diseñado se puede replicar en otras organizaciones con características similares, adecuándose a sus contextos.

Palabras clave: Modelo Sistema de Gestión, responsabilidad social, grupos de interés, gestión de calidad.

Referenciar este artículo (APA)

Díaz, A. \& Bolívar, M. (2014). Modelo de gestión estratégica de responsabilidad social organizacional. En Desarrollo Gerencial Revista de la facultada de ciencias económicas, administrativas y contables, Vol. 6 (1) P 169-195.

\footnotetext{
* Magíster en Administración de Empresas, docente de la Universidad Simón Bolívar sede Barranquilla, anadiaz1@unisimonbolivar.edu.co

* Magister en Administración de Empresas de Universidad Simón Bolívar, vinculado a Dow Química, mbolivarcordoba@dow.com
} 


\begin{abstract}
This research paper aimed to design the model of a management system, which allow improve in an organization, from the perspective of social responsibility. The analytical-interpretative method was applied, under a mixed approach of propositive nature. The sample was stratified with 178 elements (between companies and natural persons). The research techniques used were: participatory observation; surveys and interviews to employees, directors or customers. The result of this research project was the creation of a model of a management system and social responsibility, based on the theory of stakeholders, as well as the theory of the common good. It was concluded that the designed model can be repeated in other organizations with similar characteristics, being adapted to their contexts
\end{abstract}

Key word: Model of management system, social responsibility, groups of interest, quality management. 


\section{INTRODUCCIÓN}

La inquietud por la responsabilidad social organizacional se ha incrementando con el transcurrir de los años, y de manera particular en esta época, en la que fenómenos como el calentamiento global y los accidentes laborales han deteriorado la confianza de los grupos de interés en las organizaciones industriales. Para tratar el tema, se debe precisar primero que existen muchas definiciones sobre Responsabilidad Social Corporativa (RSC). Según la Comisión de las Comunidades Europeas (2006), la Responsabilidad Social Corporativa (RSC), es la acción voluntaria de las empresas por las preocupaciones sociales y medio ambientales en sus operaciones comerciales y en las relaciones con sus stakeholders. En un sentido similar Jiménez (2008) define la RSC como la implantación voluntaria de sistemas de gestión, medición e información del comportamiento socialmente responsable de una organización, considerando los intereses de los stakeholders o grupos de interés con los que la organización se relaciona. Este mecanismo constituye una forma de cumplir con ética y compromiso los requisitos exigidos por los mismos. Según Giraldo (2007), la RSC responde ante los stakeholders por los resultados que generan las decisiones adoptadas y las acciones que se desarrollan por efecto de ellas. De este modo, la responsabilidad social se constituye en un enfoque de gestión que integra toda la organización ICONTEC, (2008).

Las fundamentaciones proporcionadas por las diferentes definiciones sobre RSC muestran la 
necesidad de diseñar un modelo de gestión basado en la integración de los elementos comunes de la gestión de la calidad ambiental, seguridad y salud ocupacional, el cual debe contribuir a mejorar el desempeño de los procesos y, por ende, fomentar la confianza de los empleados y de la comunidad hacia la empresa.

Acorde con lo expuesto hasta aquí, en términos generales, el presente artículo revela información para lograr la elaboración de un modelo para un sistema de gestión de la responsabilidad social organizacional, a partir de los fundamentos teóricos del bien común y los stakeholders, tanto al interior como al exterior de la organización, a saber: empleados, directivos, propietarios, clientes, gobierno, proveedores, medio ambiente, público interesado y afectado por la acción de la empresa Izquierdo, (2005). La investigación parte de un diagnóstico que permitió conocer la situación actual de la gestión de una organización sobre las acciones de responsabilidad social, identificándose las fortalezas, debilidades, amenazas y oportunidades en la administración de los requisitos relacionados con sus clientes, empleados y la comunidad.

Los resultados de la investigación se enfocaron en la propuesta del modelo de gestión. En cada uno de los puntos de referencia de los resultados del estudio, se muestran las condiciones existentes para la implementación del modelo, recurriendo a la experiencia de la organización y a la disponibilidad asertiva del público interesado o afectado por la innovación de un estilo que también los involucra a ellos en términos de Responsabilidad Social Organizacional. 
Tras los aspectos antes descritos, es pertinente preguntarse: ¿Cómo diseñar y proponer un modelo de gestión estratégica de responsabilidad social corporativa, bajo el enfoque de los stakeholders?

\section{MÉTODO}

\section{Diseño}

Por la naturaleza de la investigación, el método utilizado es el interpretativo-analítico, Se siguió el paradigma investigativo empírico-analítico, que permite examinar detalladamente la gestión de la responsabilidad social en una organización. Bajo un enfoque mixto. Además, el estudio es propositivo, pues plantea un sistema de gestión estratégica de la responsabilidad social empresarial para una organización e integra los sistemas de gestión existentes

\section{Participantes}

La población objeto de estudio está conformada por los directivos, los clientes, los proveedores y los empleados de la organización; a mediante fórmula estadística. El resultado fue una muestra estratificada de de 178 elementos (entre empresas y personas naturales.

\section{Instrumentos}

Las técnicas de investigación utilizadas fueron: la observación participante; encuestas y entrevistas a trabajadores, directivos y clientes relacionados con la organización. La información recopilada permitió el logro de los objetivos de esta investigación

\section{Procedimientos}

La información que proviene de los documentos en los cuales se consignan los 
fundamentos teóricos, se clasifica de manera cuidadosa para su interpretación; luego se analizan los resultados a fin de extraer generalizaciones que permitan proponer un sistema estratégico de gestión de la responsabilidad social empresarial dirigido hacia los stakeholders, representados por los empleados, clientes y comunidad.

$\begin{array}{rrr}\text { Mediante } & \text { estas técnicas } & \text { se } \\ \text { reconocieron } & \text { aspectos como la }\end{array}$
infraestructura, ambiente y condiciones de la empresa objeto de estudio. También se estudió el comportamiento de los trabajadores.

Se siguieron cuatro etapas o fases en el trabajo. La primera fase se enfocó en la recuperación bibliográfica y planificación del estudio. En la segunda fase, se aplicaron las técnicas que se habían definido en el estudio; en la tercera, se diseñó el modelo de responsabilidad social; y en la última fase el modelo se sometió a validación.

\section{RESULTADOS}

En el diagnóstico realizado a la empresa objeto de investigación, se evidenció que cuenta con un sistema de gestión fundamentado por las normas de calidad ISO 9001: 2008, corroborándose su interés por entregar al mercado productos y servicios de calidad. Según Castillo \& Martínez (2006), un sistema como este se compone de ocho principios básicos: El enfoque al cliente, el liderazgo, el recurso humano, el enfoque por procesos, el enfoque sistémico, el mejoramiento continuo, la toma de decisiones basada en hechos $\mathrm{y}$ datos, $\mathrm{y}$ las relaciones mutuamente beneficiosas entre 
organización y proveedor. Así, pues, la organización estudiada basa la gestión de sus procesos en los principios antes mencionados.

En concreto, en el sistema de gestión aplicado por la organización, se identifican en un primer momento las necesidades y expectativas de los clientes. En un segundo momento, se identifican los requisitos del producto o la prestación del servicio; en el tercer momento, se entrega el producto terminado o se presta el servicio y se mantiene comunicación externa con el cliente, atendiendo incluso sus quejas y reclamos; y en el cuarto momento se retroalimenta o se toma en cuenta la percepción del cliente, en relación con la capacidad de la organización en el cumplimiento de sus necesidades y expectativas. Este sistema de gestión logró, en consecuencia, un impacto positivo en la cultura organizacional de la empresa estudiada, generando en sus trabajadores una pasión por la satisfacción de las necesidades y expectativas de sus clientes internos y externos.

Como en el caso anterior, para gestionar la seguridad y la salud ocupacional, la organización implementa la norma OHSAS 18001: 2007, la cual le permite evitar costos indirectos generados por los accidentes de trabajo, tales como el costo de reemplazo del trabajador, pérdidas de tiempo por paradas prolongadas de los bienes de la organización, y la desmotivación de los empleados ante el hecho trabajar en un ambiente inseguro.

Por otra parte, con la implementación de la norma ISO 14001: 2004, la organización busca conseguir la máxima 
calidad ambiental acorde con la situación socioeconómica de la zona en que se encuentra. Son tres los intereses perseguidos por el sistema de gestión implementado por la organización: i) prevenir la contaminación, ii) mitigar los efectos de posibles accidentes ambientales, y iii) corregir el comportamiento de los agentes socioeconómicos.

De acuerdo con los resultados, la empresa propone un estilo en la responsabilidad social desde el enfoque de stakeholders, cuyo fundamento esencial se encuentra en un sistema estratégico de gestión integrado, a partir de las experiencias obtenidas con quienes actualmente posee certificados. Para reconocer esto, fue necesario indagar sobre la percepción de la organización que tiene el público interesado, es decir, conocer la percepción con respecto a la responsabilidad social de la empresa. Estos aspectos fueron establecidos gracias a la información obtenida mediante la aplicación de encuestas a los empleados de la empresa y a los clientes y proveedores (personal directivo: jefes y coordinadores), en las cuales se propusieron factores como: Toma de decisiones, liderazgo, autonomía, desempeño, situaciones de crisis y definición de conflictos, principios empresariales, impacto, misión, visión, desarrollo organizacional y personal, comunicación, sostenibilidad, gestión, trabajo en equipo.

Desde la comprensión de la evaluación que los Clientes de la empresa hacen a la responsabilidad social de la organización, a partir de la entrevista realizada por los investigadores a 48 empresas, categorizados en dos tipos (relevancia y acuerdos), es posible 
aproximarse a los niveles de interés del público. Así, en términos cualitativos, los resultados representan la intención de reconocer el impacto de la organización objeto de estudio en la mentalidad de los clientes.

Se observó que para ellos son muy importantes las iniciativas que las empresas tienen con respecto a las acciones de responsabilidad social y que estas fortalecen los vínculos de los clientes con la organización. Además, las encuestas mostraron que un $90 \%$ de los clientes perciben el interés de la organización por capacitar a su personal en el manejo adecuado de los productos, como también por la seguridad y salud de los mismos. De igual forma, para los clientes es obvio que la empresa se preocupa por el cuidado del medio ambiente con un sello de calidad que la diferencia de la competencia.

\section{Encuesta aplicada a los trabajadores de la organización en estudio.}

Los resultados de la encuesta sobre la percepción de los trabajadores de la organización con respecto a la responsabilidad social empresarial se plasmaron en una matriz DOFA. Y al examinarla resulta evidente que la percepción de este grupo de stakeholders es muy particular. Esta percepción se presenta desde el interior de la empresa y se puede interpretar como la mirada interna de quienes son afectados de manera directa por las acciones de la organización a la cual están vinculados. 
Tabla 1. DOFA de la Organización. Sobre su gestión de responsabilidad social empresarial (RSE), a partir del modelo de los stakeholders

\begin{tabular}{|c|c|c|c|}
\hline DEBILIDADES & OPORTUNIDADES & FORTALEZAS & AMENAZAS \\
\hline $\begin{array}{l}\text { El trabajo en equipo es } \\
\text { asimilado como } \\
\text { ambiguo. } \\
\text { Persiste la idea de que } \\
\text { la empresa no se } \\
\text { responsabiliza por la } \\
\text { identificación } \\
\text { peligros en el entorno. }\end{array}$ & $\begin{array}{l}\text { La utilización } \text { de } \\
\text { estrategias para la } \\
\text { retención de talento, la } \\
\text { promoción interna y la } \\
\text { vinculación de personal } \\
\text { nuevo. } \\
\text { La RSE es una } \\
\text { herramienta de gestión. }\end{array}$ & $\begin{array}{l}\text { El reconocimiento que } \\
\text { públicamente hacen los } \\
\text { clientes y colaboradores. } \\
\text { La disponibilidad, } \\
\text { capacidad } \\
\text { conocimientos del recurso } \\
\text { humano. Las acciones } \\
\text { correctivas que se } \\
\text { implementan. }\end{array}$ & $\begin{array}{lr}\text { Los fundamentos } \\
\text { de RSE con un } \\
\text { enfoque sobre los } \\
\text { stakeholders aún } \\
\text { son débiles } \\
\text { cuestionados. }\end{array}$ \\
\hline
\end{tabular}

Fuente: Elaboración propia.

Más concretamente, desde el

enfoque de los stakeholders, la

organización considera los tres elementos

del llamado triángulo estratégico: los

intereses de la compañía (accionistas y

alta gerencia), de los colaboradores y de

los clientes; en este orden de ideas, se

puede decir que la organización., en su

estructura organizativa, incluye este

modelo en la gestión, articulado a los

mencionados tres sistemas de gestión que

se fundamentan en las normas ISO 9001:

2008, ISO 14001: 2004 y OHSAS

18001: 2007.
Basados en el enfoque de los

stakeholders, se propone, por tanto, un

modelo de gestión estratégica que asuma,

por parte de los directivos, el interés y

determinación en la destinación de

recursos y la valoración de los activos

intangibles. Asimismo, se recalca la

preocupación constante por involucrar a

la comunidad de usuarios, tanto de los

productos como de sus servicios, en el

sentido de mantener con ellos un ambiente de comunicación e información, utilizando estrategias que permitan la conectividad entre la empresa, la comunidad, las otras entidades y las 
personas con las cuales tiene relación la organización de manera directa o indirecta.

Tal como lo han expresado sus más acérrimos críticos, la filosofía y las bases del modelo con respecto a los stakeholders carecerían de fundamento si no se contaran con un respaldo epistemológico que sustente la gestión y las estrategias. En tal sentido, las bases del modelo propuesto se fundamentan en los principios establecidos por la empresa, en los resultados encontrados, en el trato que desean recibir todos los grupos de interés con relación a la continuidad, mejora en sus aspiraciones profesionales, la creación de un entorno de cooperación en su lugar de origen o en la comunidad donde desarrollan sus actividades, prosperidad y mejores condiciones de vida, entre otros. Los aspectos anteriormente enunciados son los que los clientes internos y externos esperan que la organización les ayude a alcanzar, y esto se hizo evidente con las respuestas obtenidas en las encuestas aplicadas.

Ahora bien, como ya se ha dicho, las estrategias del modelo se basan en la utilización del pensamiento estratégico, lo cual implica intuición y creatividad. Según Morrión (1994), referenciado por Urribari \& Martínez, (2009), el resultado del pensamiento estratégico es una perspectiva integrada de la empresa, en la que el verdadero cambio requiere menos reajustes de las categorías establecidas e inventar nuevas (Ramos, 2011). Es así como se deben elaborar o construir estrategias que trasciendan los cuadros mentales, como lo expresan Mintzberg \& Portuondo (1998), y que sirvan para 
alentar el aprendizaje informal que produce las nuevas perspectivas y nuevas combinaciones.

Otra herramienta es el análisis, elemento crucial del pensamiento estratégico. Pero es importante comprender al respecto que la metodología gradual y los sistemas de análisis deben ser efectivos al enfrentarse a los fenómenos y eventos del mundo real, que no siempre siguen una tendencia lineal. Por lo tanto, el medio más confiable, para dividir una situación en sus componentes y volverlos a ensamblar de acuerdo con el modelo deseado, es la utilización de un pensamiento no lineal.

Siguiendo la anterior idea, de manera organizada, se propone que la estructura y el modelo funcionen a partir de la aplicación de la metodología del 6 (seis estaciones integradas), en términos de apoyo para el conocimiento de los beneficiarios. Dicha metodología inicia con la evaluación de acciones de responsabilidad social de la organización que respondan a las pregunta de qué y cómo evaluar, teniendo como eje de las actividades, los interesados o afectados. Este proceso le permite a la empresa retroalimentarse de lo que le sucede al público afectado, así como enterarse de los cambios que se producen en el entorno para actuar según ellos.

La respuesta al qué y cómo evaluar, exige a la organización considerar la premisa básica de la administración Corporación Simón de Cirene (2007): “No podrá haber una buena gestión si no hay una evaluación de lo que le pasa a nuestro beneficiario y una retroalimentación que verifique la 
organización de acuerdo a eso que le pasó” (p. 77). El resultado de esta aplicación conllevará un aprendizaje, por lo que también se debe exigir que la información obtenida posea ciertas características que permitan usarla en la administración diaria. elementos del modelo de responsabilidad social propuesto.

.Figura 3. Modelo de gestión

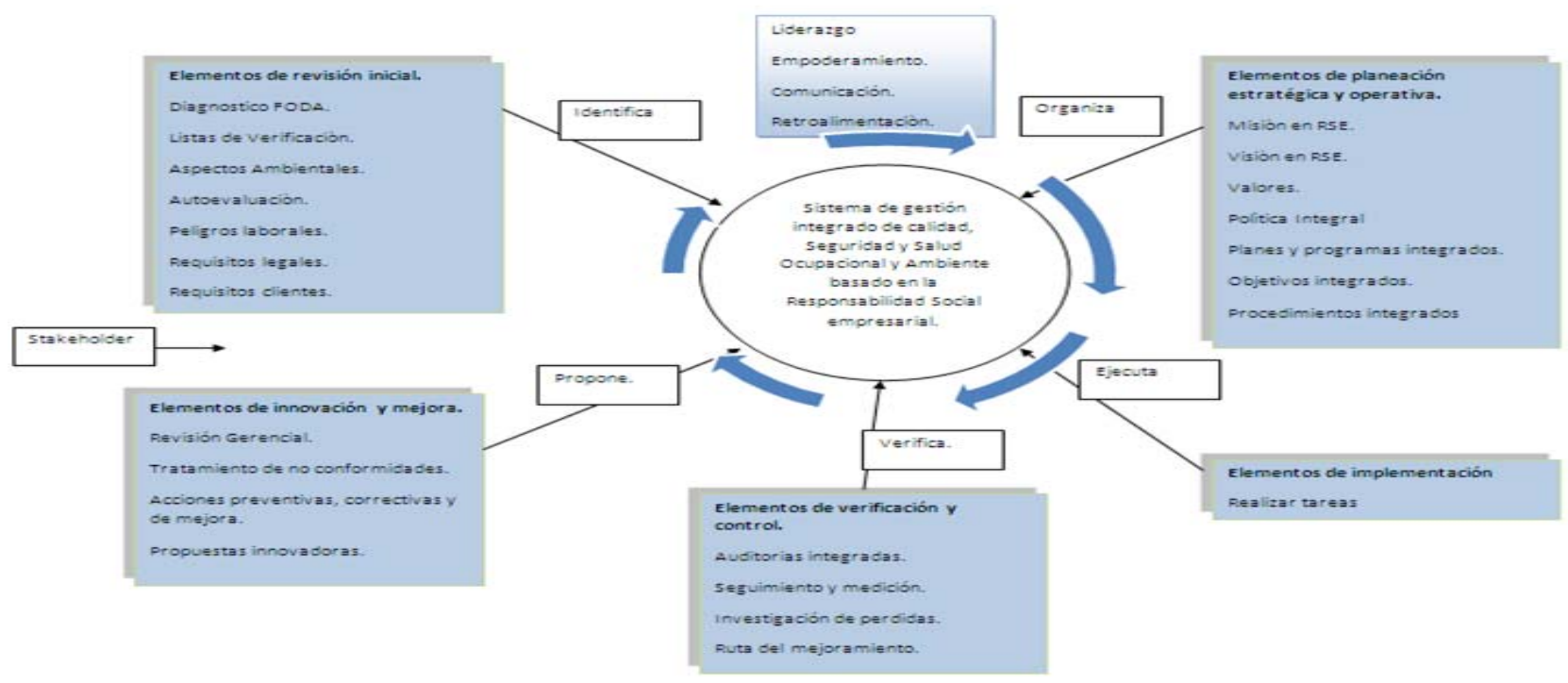

Como se observa en la figura anterior, El modelo de gestión está concebido como una rotación sucesiva de ciclos, estrategias y actividades tanto internas como externas, que confluyen en el bienestar
Común desde la base de la responsabilidad social. La rotación se inicia con la autoevaluación de los logros y dificultades de la organización. En tal sentido, la información que se recoge del entorno de clientes y público interesado es valiosa al 
momento de identificar los aspectos que le son favorables, los adversos, las oportunidades que tiene, las debilidades que le afectan, las amenazas que se ciernen y las fortalezas que la identifican.

Las actividades que se ejecutan y sistematizan en este ciclo, permiten que en un segundo ciclo de la rotación se identifique el liderazgo tanto interno como el del entorno, llevando a los directivos a tomar decisiones con respecto a: el empoderamiento en lo interno y externo de la empresa; flexibilización de los canales de comunicación y la generación de un clima de confianza en las relaciones con el público interesado.

En el ciclo de la organización se diseñan las variables que han de formar parte de la planificación estratégica orientada hacia la responsabilidad social empresarial. Por ello, se ha de revisar la estructura organizacional. Todos estos elementos se estructuran de manera integrada, de tal modo que respondan tanto a los intereses de la empresa como a las expectativas de los usuarios y del público interesado.

Lo planificado (en el ciclo de organización) se lleva a ejecución, es decir, al cumplimiento de cada una de las tareas establecidas. En este ciclo del modelo, todos los miembros de la organización, incluyendo al público interesado, tienen un compromiso que cumplir e incluso está convidado a rendir resultados.

El siguiente ciclo es el de verificación, y se asume más en función evaluativa y de retroalimentación que en términos de control y sanción. Aunque en los conceptos de este ciclo se haya consignado el componente auditoría, este se interpreta en forma integrada a los demás componentes; pues lo que se quiere con este enfoque de stakeholders en el marco de la responsabilidad social, es la permanente construcción de rutas de mejoramiento continuo. 
Por último, está el ciclo de las propuestas: Se espera que estas se implementen en forma innovadora y con una visión de conjunto, coherente con el modelo en uso.

Al terminar este último ciclo, se reinicia a partir del primero, es decir, sigue la rotación en el modelo: el proceso no se detiene, salvo que sus resultados no estén a la par de las expectativas suscitadas entre los miembros de la organización y su público interesado o afectado por las acciones de la empresa.

\section{DISCUSIÓN}

El concepto de Responsabilidad Social Empresarial nace con la noción de empresa moderna y se refiere a una nueva manera de hacer negocios, en la que las empresas tratan de encontrar un equilibrio entre la necesidad de alcanzar objetivos económicos, financieros y de desarrollo, y el impacto social o ambiental de sus actividades Rivadeneira y De Marco, (2005).

Al adoptar, entonces, una perspectiva sistémica del ambiente en sus dimensiones natural, social y económica, apremia también la incorporación de argumentos ecológicos y sociales a la planificación económica del desarrollo. Al respecto, Dixon (2010) expresa que “el más complejo reto que los negocios han enfrentado, consiste en organizarse de manera armónica con los sistemas naturales y sociales, y asegurar prosperidad a corto y a largo plazo”. Basados en estos requerimientos, en 1999, en el marco del foro económico mundial, se propuso por primera vez la creación de un pacto mundial, y a mediados del año 2000 se inició la fase operativa del mismo.

Este pacto busca aprovechar la fuerza de la acción colectiva para fomentar la responsabilidad cívica de las empresas, y así lograr que estas contribuyan a afrontar 
eficazmente los retos que plantea la globalización. De esta forma, el sector privado se vincula a la visión del Secretario General de la ONU, quien aboga por "una economía mundial más sostenible e inclusiva”. Entre los objetivos centrales del pacto destaca el de lograr que los principios y declaraciones formulados en el discurso formen parte de la estrategia y de las operaciones de las empresas, amén de facilitar la cooperación entre los principales grupos participantes.

En el caso de nuestro país, se cuenta con el documento denominado Pacto Mundial Colombia (2012). Este no es un instrumento normativo, y se basa en la responsabilidad pública, en la transparencia y en la sana defensa de los propios intereses de las empresas, para promover y ejecutar conjuntamente medidas encaminadas al logro de los principios propuestos por el Pacto Mundial, lo cual se ha convertido en un reto para las organizaciones inmersas en el nuevo sistema globalizado. En el llamado Pacto Mundial participan todos los sectores de la sociedad: los gobiernos, que definieron los principios en que se basa la iniciativa; las empresas, en cuyas actividades procura influir; las organizaciones laborales, de quienes depende directamente el proceso de la producción mundial; las organizaciones de la sociedad civil, que representan al conjunto de los grupos de interesados; y las Naciones Unidas Global Compact, (2008). Todos estos componentes constituyen al Pacto Mundial en el único foro político verdaderamente universal del mundo en lo referido a tareas de organización y facilitación.

El Pacto Mundial invita a las empresas a que hagan suyos 10 principios, los cuales están agrupados en cuatro áreas: Derechos Humanos, Medio Ambiente, Estándares Laborales y Anticorrupción.

El área de Derechos Humanos comprende dos de los principios 
mencionados: el primero procura que las empresas apoyen y respeten la protección de los derechos humanos proclamados en el ámbito internacional; el segundo demanda que las empresas se aseguren de no ser cómplices en abusos a los derechos humanos.

En el área del Medio Ambiente, las empresas deben apoyar los métodos preventivos respecto a los problemas ambientales, además de promover una mayor responsabilidad ambiental y difundir tecnologías inofensivas para el medio ambiente.

En el área de normas o estándares laborales, las organizaciones deben respetar la libertad de asociación y el reconocimiento efectivo del derecho a la negociación colectiva. También deben eliminar todas las formas de trabajo forzoso u obligatorio, abolir de forma efectiva el trabajo infantil, así como la discriminación con respecto al empleo y ocupación.
La última área comprende la lucha contra corrupción e insta a las empresas a luchar contra las diferentes formas de esta, incluyendo la extorsión y el soborno.

Otra herramienta utilizada actualmente por las organizaciones es el Balance Social. Esta parte de un trabajo iniciado en 1996 por el grupo de investigación ETHOS de la Universidad Nacional de Colombia, Sede Manizales. Con este se busca definir una norma-guía para la gestión empresarial, a partir de la medición del cumplimiento de la Responsabilidad Social Empresarial en términos de gestión social, por un periodo determinado y frente a metas preestablecidas. Lo anterior permite que la norma resulte auditable por cada empresa, con base en un modelo de balance social que busca evaluar la responsabilidad social empresarial en ocho áreas temáticas, que se dividen a su vez en una serie de variables relacionadas con la gestión social de la empresa, denominadas “variables iníciales”. 
Esta propuesta es de particular interés porque apela a la flexibilidad del modelo, ya que permite que nuevas variables e indicadores sean integrados. En general, el balance social es un instrumento estratégico que puede ser utilizado por las instituciones para evaluar, multiplicar, informar y comparar el ejercicio o impacto obtenido por las actividades o programas de responsabilidad social, tanto al interior como al exterior de las organizaciones.

El reconocimiento de la responsabilidad social como una filosofía de la organización, conlleva a que esta última estructure una política social que fije parámetros para desarrollar acciones tendientes al cumplimiento de dicha responsabilidad interna y externa. Si esta política no existe, la empresa deberá comenzar por definirla, aceptarla y respaldarla, pues de lo contrario el balance social será simplemente un informe y no una herramienta de gestión empresarial, que le

permita planificar y ejecutar acciones coherentes con su propia realidad y la de su entorno Caballero \& Balaguer,( 2006).

Otro aspecto de importancia para resaltar sobre el tema del balance social es el apoyo de la alta gerencia, debido a que sin este apoyo tal balance se convierte en un programa aislado y sin continuidad en el tiempo y, por lo tanto, no cumpliría con los objetivos propuestos de información, concertación y planificación Caballero \& Balaguer, (2006).

El último de los mecanismos
comúnmente utilizado por las
organizaciones se refiere a los Sistemas
Integrados de Gestión (SIG). Se trata de un
conjunto de elementos cuya finalidad
consiste en orientar, fortalecer, direccionar y
alinear los sistemas de gestión en forma
paralela a la dirección estratégica de la
entidad, con el fin de garantizar la eficiencia,

El último de los mecanismos (n) 
eficacia, transparencia y efectividad en la gestión organizacional. Según la Guía Nacional expedida por el DAFP y la Alcaldía Mayor de Bogotá, el sistema de gestión “es un conjunto de actividades mutuamente relacionadas que tienen por objetivo orientar y fortalecer la gestión, dar dirección, articular y alinear conjuntamente los requisitos de los subsistemas que lo componen (Gestión de Calidad, modelo estándar de control Interno, Gestión Ambiental, Gestión de Desarrollo Administrativo, entre otros)” Empresa de renovación urbana, (2013). El sistema integrado de gestión posee una estructura de árbol, con un tronco común y cuatro ramas correspondientes a las tres áreas de gestión: calidad, medio ambiente, responsabilidad social, seguridad y salud laboral. El tronco contendría el sistema de gestión común a las áreas especificadas, teniendo en cuenta todos los elementos, desde la política a la asignación de los recursos, pasando por la planificación y el control de las actuaciones, y terminando con la auditoria y la revisión del sistema.

Citando a la Real Academia Española, Herrera (1982) expresa que integrar es "formar las partes de un todo" (p. 235). Se habla de integrar sistemas, que tradicionalmente se asumen por separado en una única gestión. En este sentido, es muy deseable evitar la duplicidad de procedimientos para actividades comunes, aunque correspondan a diferentes aspectos del dinamismo de la empresa. Hoy día, muchas organizaciones han adoptado o están adoptando especificaciones y normas para sus sistemas de gestión en ámbitos diversos (calidad, medio ambiente, etc.), tales como ISO9001, ISO 14001, ISO/IEC 27001, ISO 22000, ISO/IEC 20000 y OHSAS 18001. Normalmente, estas operan como sistemas independientes, sin embargo, en todos los sistemas de gestión, hay ciertos elementos comunes que se pueden gestionar de forma integrada. De modo que la unidad esencial 
de estos sistemas en el sistema de gestión general de la organización pueda ser reconocida y utilizada de una forma más ventajosa.

A pesar de ello, solo hasta el año 2008 se desarrolló una especificación técnica, denominada PAS 99, que consiste en la “Especificación de los requisitos comunes del sistema de gestión como marco para la integración”. Esta técnica establece requisitos para la integración y combinación de los sistemas de gestión y fue desarrollada por el BSI de España (Miguel, 2013). Antes de esto no se disponía de ninguna especificación respecto a “requisitos” para sistemas de gestión integrados, pero existía el acuerdo ACX 50-200, FD ISO X 50-189, desarrollado por Dansk Standard en Dinamarca, que guiaba en el tema los países bajos Mellado (2010).

Basados en los requerimientos de la gestión, se trae a colación la norma ABNT 16001, que fue elaborada por la
ABNT/CEET (Comissão de Estudo Especial Temporária da Responsabilidade Social [Comisión Especial Temporal de Estudios de Responsabilidad Social]), y se puede aplicar a todo tipo de organizaciones que busquen demostrar desempeño ambiental, social y económico adecuado a sus stakeholders. El éxito en la implementación de esta norma depende del compromiso de todos los niveles de la organización, sobre todo de la alta dirección. El objetivo de la norma es proveer a las organizaciones de los elementos necesarios de un sistema de gestión de responsabilidad social eficaz, los cuales se puedan integrar con otros sistemas de gestión adicionales, de forma que les permita alcanzar los objetivos relacionados con sus aspectos de responsabilidad social. Este documento propone los requisitos mínimos para el establecimiento de un sistema de responsabilidad social, y permite a las organizaciones formular e implementar una política y unos objetivos coherentes con los requisitos legales $y$ otros que la 
organización suscriba con sus compromisos éticos. $\quad$ Entre estos se hallan las preocupaciones en torno a la promoción de la ciudadanía, el desempleo sustentable y la transparencia de sus actividades Holguín (2008).

Esta norma ABNT 16001 (Associação Brasileira de Normas Técnicas [Asociación Brasilera de Normas Técnicas] se fundamenta en la metodología PHVA (Planear, Hacer, Verificar, Actuar) Deming, (1982) citado por Gutiérrez (2005) y, brevemente descrita, consiste en: planear, es decir, en establecer los objetivos y procesos necesarios para que se produzcan resultados acordes con la política de responsabilidad social de la organización; hacer, que consiste en implementar los procesos; verificar, que permite monitorear y medir los procesos en relación con la política de responsabilidad social, sus objetivos, metas, requisitos legales y otros, así como en reportar sus resultados; actuar, que se concretiza en tomar decisiones para mejorar continuamente el desempeño.

Con respecto a los enfoques teóricos desde la concepción integral vinculadora del público interesado (stakeholders), desde el año 1994, la Asociación Nacional de Industriales-ANDI (2006) ha incorporado entre sus actividades los estudios relacionados con la responsabilidad social. Este interés se ha traducido en un compromiso que incluye actividades orientadas tanto a crear instituciones que favorezcan a los trabajadores y a la comunidad (el Hospital San Vicente de Paúl en Medellín, ICETEX, Cajas de Compensación Familiar, Sena, entre otras), como a formar una cultura de la responsabilidad social empresarial entre sus afiliados y los empresarios en general (Manual de balance social, código de ética, trabajo infantil y trabajo decente). En años más recientes, se creó la Gerencia Nacional de Responsabilidad Social Empresarial y se 
elaboró una encuesta específica para detectar los avances en este campo. En los resultados de la encuesta sobre Responsabilidad Social Empresarial, que fue realizada por la ANDI (2006), se encontró que prácticamente todos los empresarios encuestados (98.7\%) consideran que el sector privado debe asumir responsabilidades sociales con los trabajadores y la comunidad, más allá de las estrictamente legales.

Ahora bien, según hacia quien se dirigirá la actividad social, las actividades de responsabilidad social empresarial pueden clasificarse en dos grandes categorías: internas y externas. De las primeras hacen parte los trabajadores y accionistas. Las externas incluyen la comunidad, los usuarios y clientes, los proveedores, el gobierno y la competencia. En el manejo interno de la responsabilidad social de las empresas, la mayor prioridad está en los trabajadores, quienes tienen la mayor cercanía con la empresa; mientras que en lo externo, la comunidad ocupa el primer lugar, teniéndose en cuenta el impacto que esta tiene sobre la actividad diaria de las empresas ANDI (2011).

En América Latina y en el entorno de las empresas españolas con presencia en el continente, la responsabilidad social empresarial aplicada a cada una de las acciones y estrategias que la organización define con la inclusión del público interesado, ha sido percibida desde diferentes enfoques y modelos. En el campo de las relaciones públicas, una muestra de ello es la que aparece consignada en el informe La responsabilidad social corporativa de las grandes empresas españolas con presencia en América Latina: modelos de actuación, de la autoría de Polo (2009), del cual destacamos el siguiente fragmento:

“En términos generales, la idea de la relación con las audiencias permea y domina 
la vigente práctica de los profesionales de la comunicación y las relaciones públicas. La dimensión que la Responsabilidad Social Corporativa tiene en la comunicación es una aplicación evidente de estos criterios o principios que pretenden medir y hacer sólidas las relaciones de las empresas con sus stakeholders sean estos internos 0 externos, se llamen empleados, accionistas, proveedores, medios, autoridades o sociedad” (p. 27).

De todos modos, la teoría de Freeman (1964), en su modelo de stakeholders, se puede considerar la de mayor madurez conceptual. Posiblemente, en la misma aplicación, la característica de los stakeholders se hace extensiva a los empleados, clientes, proveedores, distribuidores, competidores y comunidades. De acuerdo con la interpretación de Polo (2009), esta teoría “sitúa el término participante como un individuo o grupo que puede afectar o verse afectado por el logro de los objetivos de una firma” (pp. 92-93).

Si la conceptualización en tal sentido es aplicada a una empresa en específico, la connotación de Freeman resulta válida y al mismo tiempo responde a un giro sustancial en la visión axial de la misma desde los accionistas y gestores. La empresa ya no se dirige, entonces, para el beneficio de unos pocos, sino pensando en el bien de públicos amplios, diversos y, a veces, con intereses distintos. Como se puede ver, la teoría de Freeman sobre los stakeholders o grupos de interés representa en el fondo un esquema de gestión de las organizaciones y un planteamiento de ética empresarial.

En este sentido, las empresas son un lugar de muy complejas relaciones, donde se encuentran intereses distintos, a veces enfrentados, que los propietarios, gestores o directores deben tener en cuenta para encontrar un punto de equilibrio entre la 
propiedad, el trabajo, la comunidad, el consumidor y la política. Vemos así la importancia de que los gestores y directivos cumplan con su participación en el proceso.

Lo expuesto anteriormente se complementa con lo que Garriga y Melé (2004) consideran sobre diferentes aproximaciones a la Responsabilidad Social Corporativa (RSC), que según el punto de vista de los autores mencionados y de acuerdo con la interpretación de Toro (2006), está soportada por “diferentes enfoques que llegan a ser contradictorios, complejos y pocos claros” (p. 339). Esto obliga a formular una especie de cartografía, desde el punto de vista metodológico, de las principales teorías que de alguna manera se aproximan a la práctica de la responsabilidad social de las empresas. Desde esta perspectiva, se puede contar con tres grupos de teorías. El primero se relaciona con el enfoque instrumental correspondiente a las prácticas de las empresas, que apuestan por un comportamiento ético y responsable en la medida en que tal comportamiento aporte ventajas competitivas al negocio. El segundo grupo se enfoca en las fundamentaciones teóricas que se centran en el poder de las corporaciones en la sociedad, y en el uso responsable de este poder en el plano político. El tercero lo conforman las teorías correspondientes a la satisfacción, por parte de la empresa, de las demandas sociales de sus stakeholders.

Estos enfoques sirvieron de fundamento para la aplicación y obtención de los resultados de la presente investigación, permitiéndoles mantener un equilibrio entre los objetivos económicos, ambientales y sociales, y lográndose así un acercamiento a las visiones que conforman las teorías integradas, o sea, la de la gestión de las demandas de los stakeholders o grupo de interés de la empresa. 
De manera intencional o siguiendo el contenido de la literatura sobre el tema, la responsabilidad social organizacional se califica como un proceso o programa que está en la agenda de muchos empresarios. Aun cuando empezó como una moda, al menos ha sido una moda perdurable que debe aprovecharse para, primero, adecuarla al entorno y a la mentalidad de directivos empresariales, empleados, clientes y demás público interesado en las actividades de la organización; y, segundo, implementarla con modelos de gestión que enfaticen o tomen como parámetros uno o dos de los componentes fundamentales de la empresa.

El modelo de gestión de responsabilidad social organizacional que se ha propuesto, se apoya en el concepto de los stakeholders, con fundamento en la teoría del bien común, debido a la importancia de conocer la percepción que cada uno de los actores tiene sobre las acciones de la
La responsabilidad social organizacional no es una simple moda, como se ha querido hacer ver por parte de quienes han criticado fuertemente su práctica, más aún si sobre ella se ajustan o introducen ciertas metodologías de apoyo que de alguna manera la extiendan, de la obligación de la empresa, a los deberes de los usuarios o grupos de interés de esta organización, como ha ocurrido en los países de Europa, los Estados Unidos y algunos de América Latina. Los enfoques actuales relacionados con responsabilidad social organizacional se enfatizan en la búsqueda de puntos de convergencia de intereses, de tal manera que los beneficios económicos y sociales no solo lleguen a los inversionistas, los propietarios de las empresas o los directivos, sino que también toquen las expectativas de los usuarios, beneficiarios, clientes (internos y externos), la comunidad $\mathrm{y}$, en última instancia, la sociedad del planeta, si se organización. 
quiere lograr un verdadero desarrollo sostenible.

El enfoque de responsabilidad social organizacional, apoyado en la teoría de los stakeholders (grupos interesados) y en la teoría del bien común, es una alternativa que traslada de manera integral la participación en la gestión de la empresa, aprovechando lo que Mintzberg (1994) ha expuesto sobre la importancia del diseño organizacional para el desarrollo de las funciones de una empresa. Todo ello en pro de una organización efectiva, que logre coherencia entre sus componentes y, al mismo tiempo, brinde la posibilidad de replicar su modelo en otras organizaciones con características similares.

\section{REFERENCIAS}

Asociación Nacional de Empresarios de Colombia. ANDI (2011). En: andiinformeencuesta_responsabilidadsocial.pdf
Asociación Nacional de Empresarios de Colombia. ANDI. (2006). Encuesta sobre Responsabilidad Social Empresarial (Consultado en 2011 en: andi-informeencuesta_responsabilidadsocial.pdf

Caballero, I. \& Vega, B (2006). NTP 687: Responsabilidad social de las empresas: Modelo de Balance Social de ANDI - OIT (I) ministerio de trabajo y asuntos sociales España. http://www.mapfre.com/documentaci on/publico/i18n/catalogo_imagenes/g rupo.cmd?path=1031485

Castillo, D. y J. Martínez. (2006). Enfoque para combinar e integrar la gestión de sistemas. Bogotá: ICONTEC.

Comisión de las comunidades europeas (2006). Poner en práctica la asociación para el crecimiento y el empleo: hacer de Europa un polo de excelencia de la responsabilidad social de las empresas. Bruselas

Corporación Simón de Cirene. (2007). Manual para la administración de Empresas Sociales. (2a. Ed.). Santiago de Chile. Extraído el 23 de 
Julio de 2011 desde

www.simondecirene.cl

Dixon, F. (2010) Total Corporate Responsibility. Achieving Sustainability and Real Prosperity. In: Ethical Corporation Magazine, 1 (4)

Empresa de renovación urbana (ERU) (2013). Sistema integrado de gestión SIG alcaldía mayor de Bogotá D.C. http://www.eru.gov.co/contenido/nos otros/235-sistema-integrado-degestion-sig

Freeman, E. (1964). Strategic Management: A Stakeholder Approach http://www.un.org/es/globalcompact/ context.shtml.

Giraldo, C. (2007). ¿¿Protección o desprotección social? Bogotá: Universidad Nacional de Colombia.

Global Compact (2008). A global initiativeA global agenda, Naciones Unidas, Documento de Internet. [Acceso: 21 noviembre 2009]
Gutiérrez, A. (2005). Aplicación de los círculos de calidad en una organización universidad Autónoma de Hidalgo Pachuca.

Herrera, F. (1982). "Presencia de Bello en la integración cultural latinoamericana” Homenaje a Don Andrés Bello. Santiago: jurídica de Chile.

Holguín N. (2008). Guía técnica Colombiana sobre Responsabilidad social en consulta pública comunicado de prensa INCONTEC Colombia

Instituto Colombiano de Normas Técnicas y Certificación (ICONTEC) (2008). Guía técnica Colombiana (GTC 180). I.C.S 03.100.99. Bogotá D.C.

Jiménez R. (2008). Reflexiones sobre la teoría de la responsabilidad social empresarial: una mirada desde la contabilidad. Universidad de Manizales. VII Simposio Nacional de 
Investigación Contable y Docencia. Universidad Nacional de Colombia.

Mellado, M. (2006). La Gestión Integrada de la Calidad. Buenos Aires: Ramón Areces.

Miguel, J. (2013). Pas 99 Especificación de los requisitos comunes del sistema de gestión como marco para la integración http://www.aec.es/c/document_librar y/get_file?uuid=d4f00264-ee744abd-b1c84e48fccd6836\&groupId=10128
Pacto global Colombia (2012). Visión desde la óptica de la responsabilidad social http://www.slideshare.net/yulderjime nez/pacto-global-y-responsabilidadsocial

Polo Martín, J. F, (2009). La responsabilidad social corporativa de las grandes empresas españolas con presencia en América Latina: modelos de actuación. Memoria de Tesis Doctoral. Universidad Complutense de Madrid. Facultad de Ciencias de la Información. Madrid.
Rivadeneira, M. De Marco, M. (2005). Balance social: una medida de la responsabilidad social empresaria. Tucumán, Argentina: Universidad del Norte Santo Tomás de Aquino
Ramos, M. (2011). La caída y el ascenso de la planificación estratégica. http://es.scribd.com/doc/51173418/ca idascenso-de-la-planeacionestretegica-mintzberg
Mintzberg, H. \& Vélez, O. (1998). La caída y el ascenso de la planificación estratégica. Harvard Bussines review (4-18)
Server, R. J. y Villalonga, G. (2005). “La Responsabilidad Social Corporativa (RSC) y su gestión integrada”. CIRIEC-España, Revista de 
economía pública, social y cooperativa, 53): 137-161.

Server Izquierdo, R. \& Villalonga G. I. (2005). La responsabilidad Social Corporativa (RSC) y su gestión integrada, Economía Pública, Social y Cooperativa. Revista de economía pública, social y cooperativa, (53), 137-161.

Toro, D. (2006). El enfoque estratégico de la responsabilidad social corporativa: revisión de la literatura académica. En: Intangible Capital. 2(14), 338358

Urribari, A., \& Martínez, F. (2009). Elementos del pensamiento estratégico que fortalecen la comunicación organizacional Revista electrónica facultad de Ingeniería UVM 3(2) Universidad Valle de Monterrey. 